\title{
Active Cancer and Elevated D-Dimer Are Risk Factors for In-Hospital Ischemic Stroke
}

\author{
Izumi Yamaguchi ${ }^{a}$ Yasuhisa Kanematsu ${ }^{a}$ Kenji Shimada ${ }^{a}$ \\ Masaaki Korai $^{a}$ Takeshi Miyamoto $^{a}$ Eiji Shikata ${ }^{a}$ Tadashi Yamaguchi ${ }^{a}$ \\ Nobuaki Yamamoto ${ }^{b}$ Yuki Yamamoto $^{b}$ Keiko T. Kitazato ${ }^{a}$ \\ Yoshihiro Okayamac ${ }^{c}$ Yasushi Takagia \\ a Department of Neurosurgery, Tokushima University Graduate School of Biomedical \\ Sciences, Tokushima, Japan; ${ }^{b}$ Department of Clinical Neuroscience, Tokushima University \\ Graduate School of Biomedical Sciences, Tokushima, Japan; ${ }^{\circ}$ Clinical Trial Center for \\ Development Therapeutics, Tokushima University Hospital, Tokushima, Japan
}

Keywords

In-hospital stroke $\cdot$ Cancer $\cdot$ D-dimer $\cdot$ Fibrinogen $\cdot$ Hypercoagulability

\begin{abstract}
Background and Purpose: Little attention has been paid to the pathogenesis of in-hospital stroke, despite poor outcomes and a longer time from stroke onset to treatment. We studied the pathophysiology and biomarkers for detecting patients who progress to in-hospital ischemic stroke (IHS). Methods: Seventy-nine patients with IHS were sequentially recruited in the period 2011-2017. Their characteristics, care, and outcomes were compared with 933 patients who had an out-of-hospital ischemic stroke (OHS) using a prospectively collected database of the Tokushima University Stroke Registry. Results: Active cancer and coronary artery disease were more prevalent in patients with IHS than in those with OHS (53.2 and 27.8\% vs. 2.0 and $10.9 \%$, respectively; $p<0.001$ ), the median onset-to-evaluation time was longer (300 vs. $240 \min ; p=0.015$ ), and the undetermined etiology was significantly higher (36.7 vs. 2.4\%; $p<0.001$ ). Although there was no significant difference in stroke severity at onset between the groups, patients with IHS had higher modified Rankin Scale (mRS) scores (3-6) at discharge (67.1 vs. $50.3 \% ; p=0.004)$ and rates of death during hospitalization $(16.5$ vs. $2.9 \% ; p<$ $0.001)$. D-dimer (5.8 vs. $0.8 \mu \mathrm{g} / \mathrm{mL} ; p<0.001$ ) and fibrinogen (532 vs. $430 \mathrm{mg} / \mathrm{dL} ; p=0.014$ ) plasma levels at the time of onset were significantly higher in patients with IHS after propensity score matching. Multivariate logistic regression analysis revealed that active cancer (odds ratio $[\mathrm{OR}] 2.30 ; 95 \%$ confidence interval $[\mathrm{Cl}]$ 1.26-4.20), prestroke mRS scores 3-5 (OR 6.78;
\end{abstract}


95\% $\mathrm{Cl} 3.96-11.61$ ), female sex (OR 1.57; 95\% Cl 1.19-2.08), and age $\geq 75$ years (OR 2.36; 95\% $\mathrm{Cl}$ 1.80-3.08) were associated with poor outcomes. Conclusions: Patients with IHS had poorer outcomes than those with OHS because of a higher prevalence of active cancer and functional dependence before stroke onset. Elevated plasma levels of D-dimer and fibrinogen, especially with active cancer, can help identify patients who are at a higher risk of progression to IHS.

(C) 2019 The Author(s)

Published by S. Karger AG, Basel

\section{Introduction}

In-hospital ischemic stroke (IHS) is an acute stroke in patients admitted to hospital with another diagnosis or for a different procedure. These patients present with distinct risk factors and worse outcomes than out-of-hospital ischemic stroke (OHS) patients [1]. An estimated 2.2-17\% of acute strokes are of in-hospital onset [2]. However, less attention has been paid to the pathogenesis of IHS, despite poor outcomes and the longer time from stroke onset to treatment.

Many stroke patients have been treated at our stroke center, established in 2005, with various therapeutic options, including patients with IHS and OHS. Their data, including characteristics, care, and outcomes, are compiled by the Tokushima University Stroke Registry. We believe that it is crucial to clarify the differences between patients with IHS and OHS, to be able to develop strategies to reduce the incidence of IHS.

Several studies have examined the prognostic and diagnostic implications of elevated D-dimer as a risk factor for stroke in patients with cancer and cardiovascular diseases [3-6]. Although plasma D-dimer levels reflect fibrin turnover and are a unique thrombosis biomarker, levels that predict ischemic stroke are unknown. Stroke is often observed in patients with cancer or cardiovascular disease and is a devastating event with high mortality; however, predictors of mortality in patients with IHS who have a history of cancer and/or cardiovascular disease are poorly elucidated. Furthermore, the timing of IHS onset remains unclear.

We examined characteristics, care, and outcomes in patients with IHS. These data were compared to the same parameters measured in patients with OHS. We also investigated the pathophysiology of IHS and biomarkers for the early detection of patients at a high risk of progression to IHS.

\section{Materials and Methods}

\section{Patient Recruitment and Characteristics}

This retrospective analysis used prospectively collected stroke registry data obtained from consecutive patients admitted and treated for ischemic stroke in the Stroke Center at Tokushima University Hospital between January 2011 and December 2017. We determined stroke subtypes using 12 channel electrocardiograms, 24-h electrocardiogram monitoring, echocardiography, carotid ultrasonography, and brain magnetic resonance imaging. Figure 1a shows the case-selection flowchart of the study. Among 1,178 ischemic stroke patients, those with a transient ischemic attack $(n=124)$, IHS at another hospital and subsequently admitted to our hospital ( $n=31$ ), IHS at the Department of Neurology/Neurosurgery $(n=7)$, and those with insufficient data to distinguish IHS from OHS $(n=4)$ were excluded from this study. In total, 1,012 ischemic stroke patients were finally included in this study. These patients were divided into 2 groups: the IHS group, i.e., when the stroke occurred in hospital, 
and the OHS, i.e., when the stroke occurred outside the hospital. We conducted betweengroup comparisons of demographics, past medical history, medications taken before stroke, the prestroke modified Rankin Scale (mRS) score, the National Institutes of Health Stroke Scale (NIHSS) score at stroke onset, onset-to-evaluation (OTE) time, the stroke subtype using the Trial of ORG 10172 in Acute Stroke Treatment (TOAST) classification [7], mRS score at discharge, mortality during hospitalization, and the levels of plasma D-dimer and fibrinogen.

The OTE time was defined as the time point of stroke physician evaluation minus the time point of stroke onset. Stroke onset times were decided as the time the patient was last known to be well or that the onset of symptoms was noticed. A poor outcome was defined as an mRS score of 3-6 at discharge, or in-hospital death. Primary diseases for the admission of patients with IHS were classified using the International Statistical Classification of Diseases and Related Health Problems, Tenth Revision (ICD-10) coding of disease entities [8]. All cancers were staged using the tumor-node-metastasis (TNM) classification system from the Union for International Cancer Control (UICC) for solid cancers or the Ann Arbor classification for malignant lymphoma $[9,10]$. Active cancer was defined as cancer diagnosed within 6 months before stroke onset, any treatment for cancer within the previous 6 months, or recurrent or metastatic cancer [11]. Patients with cancer-related ischemic strokes were those with (a) a stroke of undetermined etiology, (b) active cancer, and (c) elevated D-dimer levels ( $\geq 1.0 \mu \mathrm{g} /$ $\mathrm{mL}$ ).

Plasma levels of coagulation markers, including D-dimer, were elevated in patients with acute ischemic stroke and cancer or cardiovascular diseases $[3,4,12,13]$. These findings might reflect the hypercoagulable state of stroke patients. The plasma levels of D-dimer and fibrinogen within $72 \mathrm{~h}$ of stroke onset were compared between patients with IHS and OHS after propensity score-matching to adjust for age, sex, hypertension, diabetes mellitus, dyslipidemia, and atrial fibrillation (AF).

\section{Statistical Analysis}

Comparisons were performed using the Wilcoxon rank-sum test for continuous variables and the $\chi^{2}$ test for categorical variables. Univariate and multivariate logistic regression analyses were performed to identify factors associated with poor outcomes (mRS 3-6 at discharge). The stepwise forward selection method was used to select the variables for multivariate analysis. The $p$ value threshold for entry was set at 0.05 . Propensity score-matching was conducted to adjust for age, sex, hypertension, diabetes mellitus, dyslipidemia, and AF between patients with IHS and OHS. All statistical analyses were performed using JMP ${ }^{\circledR} 13$ (SAS Institute Inc., Cary, NC, USA) and $p<0.05$ was considered statistically significant.

\section{Results}

Differences in Baseline Characteristics and Care between Patients with IHS and OHS

We examined the data on 1,012 patients with ischemic stroke, 79 of whom (7.8\%) had IHS, and 933 of whom (92.2\%) had OHS, similar to a previous report [2]. Patients with IHS were younger (mean age 68.5 vs. 72.7 years; $p=0.006$ ), and more likely to have coronary artery disease (27.8 vs. $10.9 \% ; p<0.001$ ) and active cancer (53.2 vs. $2.0 \% ; p<0.001$ ) than patients with OHS (Table 1). In IHS patients, prestroke mRS 3-5 (functional dependence) was higher than in OHS patients $(30.4$ vs. $12.4 \% ; p<0.001)$, mostly due to the primary disease condition. There were no significant between-group differences regarding the use of antithrombotic agents and statins or stroke severity. Importantly, patients with IHS had a significantly longer OTE time than OHS patients (median 300 vs. $240 \mathrm{~min} ; p=0.015$ ). Although 
Yamaguchi et al.: Progression to In-Hospital Ischemic Stroke

Table 1. Baseline characteristics and care between patients with IHS and OHS

\begin{tabular}{|c|c|c|c|}
\hline & $\begin{array}{l}\text { IHS } \\
(n=79)\end{array}$ & $\begin{array}{l}\text { OHS } \\
(n=933)\end{array}$ & $p$ value \\
\hline \multicolumn{4}{|l|}{ Demographics } \\
\hline Male sex & $51(64.6)$ & $577(61.8)$ & 0.632 \\
\hline Age, years $($ mean \pm SD) & $68.5 \pm 13.0$ & $72.7 \pm 12.8$ & $0.006^{*}$ \\
\hline \multicolumn{4}{|l|}{ Medical history } \\
\hline Hypertension & $46(58.2)$ & $630(67.5)$ & 0.098 \\
\hline Diabetes mellitus & $26(32.9)$ & $257(27.5)$ & 0.315 \\
\hline Dyslipidemia & $14(17.7)$ & 157 (16.8) & 0.840 \\
\hline Atrial fibrillation & $15(19.0)$ & $155(16.6)$ & 0.593 \\
\hline Stroke/TIA & $13(16.5)$ & $155(16.6)$ & 0.971 \\
\hline Smoking & $31(39.2)$ & $373(40.0)$ & 0.898 \\
\hline Hemodialysis & $1(1.3)$ & $6(0.6)$ & 0.562 \\
\hline Coronary artery disease & $22(27.8)$ & $102(10.9)$ & $<0.001^{*}$ \\
\hline Active cancer & $42(53.2)$ & $19(2.0)$ & $<0.001^{*}$ \\
\hline \multicolumn{4}{|l|}{ Medications before stroke } \\
\hline Antiplatelet agent & $19(24.1)$ & 212 (22.7) & 0.788 \\
\hline Anticoagulant agent & $13(16.5)$ & 111 (11.9) & 0.254 \\
\hline Statin pretreatment & $10(12.7)$ & $128(13.7)$ & 0.790 \\
\hline \multicolumn{4}{|l|}{ Prestroke mRS score } \\
\hline $0-2$ & $55(69.6)$ & $817(87.6)$ & $<0.001^{*}$ \\
\hline $3-5$ & $24(30.4)$ & $116(12.4)$ & $<0.001^{*}$ \\
\hline NIHSS score at stroke onset & $6(2-16)$ & $4(2-12)$ & 0.127 \\
\hline & $(n=67)$ & $(n=923)$ & \\
\hline Onset-to-evaluation time & $300(113-1,440)$ & $240(90-540)$ & $0.015^{*}$ \\
\hline & $(n=74)$ & $(n=775)$ & \\
\hline \multicolumn{4}{|l|}{ Type of treatment } \\
\hline Intravenous thrombolysis & $5(6.3)$ & 109 (11.7) & 0.121 \\
\hline Endovascular therapy & $11(13.9)$ & $124(13.3)$ & 0.874 \\
\hline
\end{tabular}

Values are expressed as $n(\%)$ or median (interquartile range), unless otherwise specified. IHS, in-hospital ischemic stroke; OHS, out-of-hospital ischemic stroke; TIA, transient ischemic attack; mRS, modified Rankin Scale; NIHSS, National Institutes of Health Stroke Scale. ${ }^{*} p<0.05$, statistically significant.

there was no significant difference between the 2 groups, patients with IHS tended to receive intravenous thrombolytic therapy less often than patients in the OHS group (6.3 vs. $11.7 \%$; $p=0.121$ ). The rate of endovascular therapy was not significantly different between the groups (13.9 vs. $13.3 \%$; $p=0.874)$.

\section{Most Patients with IHS Had Neoplasms or a Disease of the Circulatory System at Admission}

Neoplasms (53.2\%) were the most prevalent primary disease in patients with IHS (online suppl. Table 1; for all online suppl. material, see www.karger.com/doi10.1159/000504163). Approximately $80 \%$ of patients with IHS had neoplasms (Chapter II, C00-D48) or diseases of the circulatory system (Chapter IX, I00-I99). The most frequent cancer type was lung cancer (35.7\%) (online suppl. Table 2). Histologically, the frequency of adenocarcinoma was high (59.5\%). Patients with active cancers were primarily diagnosed with advanced-stage disease and $81.0 \%$ of them were stage III or IV disease. 

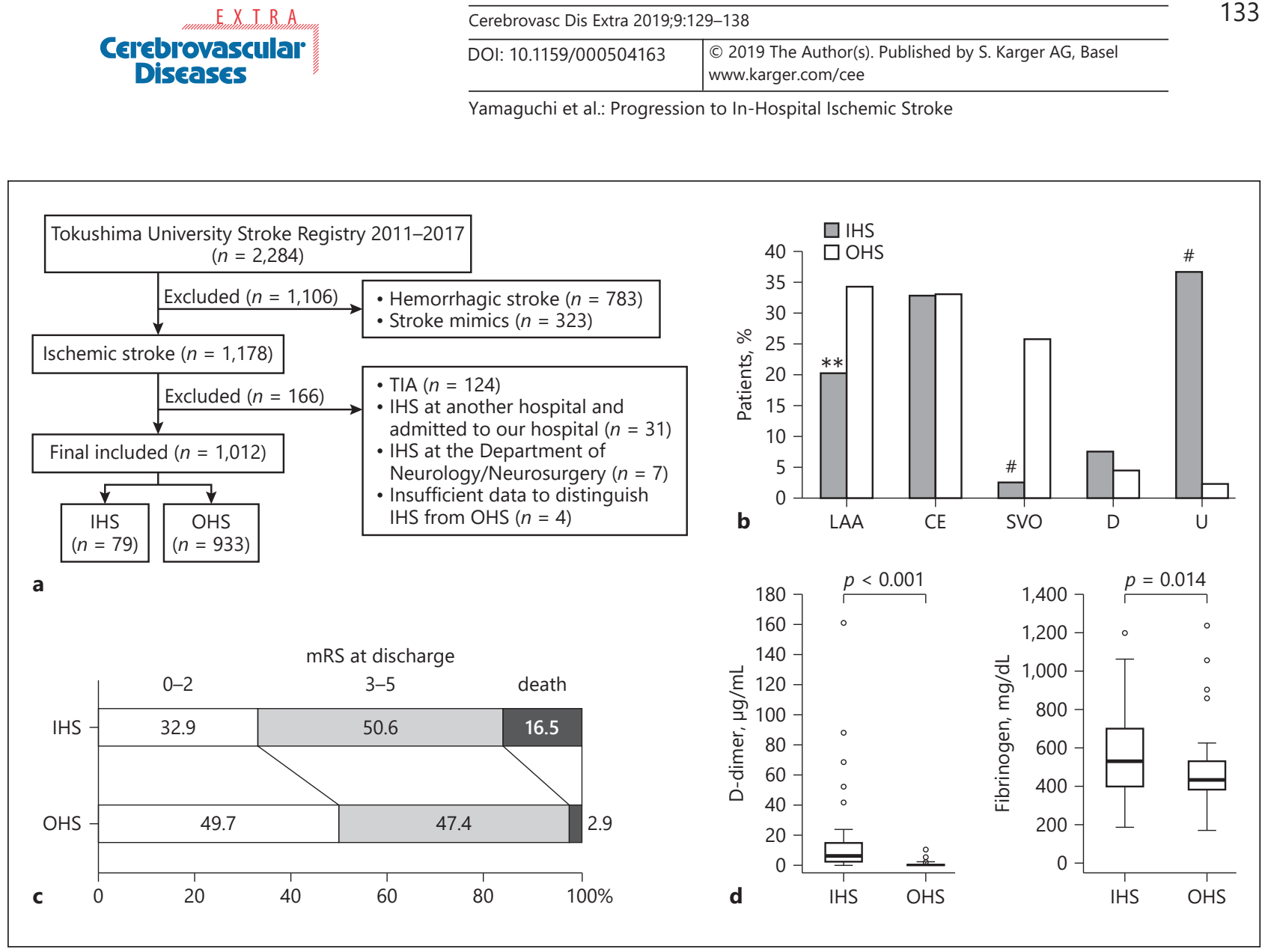

Fig. 1. a Case-selection flowchart. b Stroke subtypes in patients with IHS and OHS. c mRS at discharge in patients with IHS and OHS. d Left: D-dimer levels in patients with IHS ( $n=56$; median 5.8; IQR 2.3-15.1) and OHS ( $n=56$; median 0.8; IQR 0.5-1.2). Right: fibrinogen levels in patients with IHS ( $n=56$; median 532; IQR 395-698) and OHS ( $n=56$; median 430; IQR 381-528). IHS, in-hospital ischemic stroke; OHS, out-of-hospital ischemic stroke; TIA, transient ischemic attack; LAA, large-artery atherosclerosis; CE, cardioembolism; SVO, small-vessel occlusion; D, stroke of determined etiology; U, stroke of undetermined etiology; mRS, modified Rankin Scale. ${ }^{* *} p<0.01 ;{ }^{*} p<0.001$.

\section{Patients with IHS and Active Cancers Had Poorer Outcomes}

Rates of large-artery atherosclerosis and small-vessel occlusion were lower in IHS patients than in OHS patients (20.3 vs. $34.3 \%$ [ $p=0.008]$ and 2.5 vs. $25.7 \%[p<0.001]$, respectively) (Fig. 1b). There were no significant between-group differences for cardioembolism or stroke of determined etiology. Interestingly, there was a markedly higher prevalence of stroke of undetermined etiology in IHS patients than in OHS patients (36.7 and 2.4\%, respectively; $p<0.001)$. Twenty-two of the 42 patients with IHS (52.4\%) with active cancer were registered as having cancer-related ischemic stroke, indicating the importance of cancer in the pathology of IHS. Functional impairment at discharge, as assessed by the mRS, trended toward poorer outcomes in patients with IHS (Fig. 1c). mRS scores of 3-6 at discharge (67.1 IHS vs. 50.3\% OHS; $p=0.004$ ) and mortality during hospitalization (16.5 IHS vs. $2.9 \%$ OHS, $p<0.001$ ) were significantly higher in patients with IHS, suggestive of poorer outcomes. Multivariate logistic regression analysis revealed that female sex (odds ratio [OR] 1.57; 95\% confidence interval [CI] 1.19-2.08), age $\geq 75$ years (OR 2.36; 95\% CI 1.80-3.08), active cancer (OR 2.30; 95\% CI 1.26-4.20), and prestroke mRS 3-5 (OR 6.78; 95\% CI 3.96-11.61) were independent factors associated with poorer outcomes (Table 2). Statin pretreatment (OR $0.61 ; 95 \%$ CI $0.41-0.91$ ) was a protective factor for favorable outcomes. Importantly, outcomes were not related to in-hospital onset per se, but to a patient's background. 
Yamaguchi et al.: Progression to In-Hospital Ischemic Stroke

Table 2. Multivariate logistic regression analysis model of the probability of a poor outcome, i.e. an mRS score of 3-6 at discharge

\begin{tabular}{|c|c|c|c|c|c|c|c|c|}
\hline \multirow[t]{2}{*}{ Factors } & \multicolumn{2}{|c|}{$\begin{array}{l}\text { Patients with an } \\
\text { mRS score } 3-6\end{array}$} & \multicolumn{3}{|c|}{ Univariate analysis } & \multicolumn{3}{|c|}{ Multivariate analysis } \\
\hline & $n$ & $\%$ & OR & $(95 \% \mathrm{CI})$ & $p$ value & OR & $(95 \% \mathrm{CI})$ & $p$ value \\
\hline \multicolumn{9}{|c|}{ In-hospital onset } \\
\hline No & 933 & 50.3 & 1 (ref.) & - & & & & \\
\hline Yes & 79 & 67.1 & 2.02 & $(1.24-3.28)$ & $0.004^{*}$ & & & \\
\hline \multicolumn{9}{|l|}{ Gender } \\
\hline Male & 628 & 45.9 & 1 (ref.) & - & & 1 (ref.) & - & \\
\hline Female & 384 & 60.9 & 1.84 & $(1.42-2.38)$ & $<0.001^{*}$ & 1.57 & $(1.19-2.08)$ & $0.002^{*}$ \\
\hline \multicolumn{9}{|l|}{ Age } \\
\hline$<75$ years & 513 & 39.4 & 1 (ref.) & - & & 1 (ref.) & - & \\
\hline$\geq 75$ years & 499 & 64.1 & 2.75 & $(2.13-3.55)$ & $<0.001^{*}$ & 2.36 & $(1.80-3.08)$ & $<0.001^{*}$ \\
\hline \multicolumn{9}{|l|}{ Hypertension } \\
\hline No & 336 & 50.6 & 1 (ref.) & - & & & & \\
\hline Yes & 676 & 52.1 & 1.06 & $(0.82-1.38)$ & 0.658 & & & \\
\hline \multicolumn{9}{|c|}{ Diabetes mellitus } \\
\hline No & 729 & 52.3 & 1 (ref.) & - & & & & \\
\hline Yes & 283 & 49.8 & 0.91 & $(0.69-1.19)$ & 0.486 & & & \\
\hline \multicolumn{9}{|l|}{ Dyslipidemia } \\
\hline No & 841 & 53.2 & 1 (ref.) & & & & & \\
\hline Yes & 171 & 43.9 & 0.69 & $(0.49-0.96)$ & $0.027^{*}$ & & & \\
\hline \multicolumn{9}{|c|}{ Atrial fibrillation } \\
\hline No & 842 & 49.4 & 1 (ref.) & - & & & & \\
\hline Yes & 170 & 62.4 & 1.7 & $(1.21-2.38)$ & $0.002^{*}$ & & & \\
\hline \multicolumn{9}{|c|}{ A history of stroke/TIA } \\
\hline No & 844 & 52.7 & 1 (ref.) & - & & & & \\
\hline Yes & 168 & 45.8 & 0.76 & $(0.54-1.06)$ & 0.103 & & & \\
\hline \multicolumn{9}{|c|}{ A smoking history } \\
\hline No & 608 & 56.4 & 1 (ref.) & - & & & & \\
\hline Yes & 404 & 44.3 & 0.61 & $(0.48-0.79)$ & $<0.001^{*}$ & & & \\
\hline \multicolumn{9}{|c|}{ Coronary artery disease } \\
\hline No & 888 & 50.6 & 1 (ref.) & - & & & & \\
\hline Yes & 124 & 58.9 & 1.4 & $(0.96-2.05)$ & $0.002^{*}$ & & & \\
\hline \multicolumn{9}{|l|}{ Active cancer } \\
\hline No & 951 & 50.4 & 1 (ref.) & - & & 1 (ref.) & - & \\
\hline Yes & 61 & 70.5 & 2.35 & $(1.34-4.14)$ & $0.002^{*}$ & 2.30 & $(1.26-4.20)$ & $0.007^{*}$ \\
\hline \multicolumn{9}{|c|}{ Antiplatelet agent } \\
\hline No & 781 & 52.1 & 1 (ref.) & - & & & & \\
\hline Yes & 231 & 49.8 & 0.91 & $(0.68-1.22)$ & 0.534 & & & \\
\hline \multicolumn{9}{|c|}{ Anticoagulant agent } \\
\hline No & 888 & 50.3 & 1 (ref.) & - & & & & \\
\hline Yes & 124 & 60.5 & 1.51 & $(1.03-2.22)$ & $0.034^{*}$ & & & \\
\hline \multicolumn{9}{|c|}{ Statin pretreatment } \\
\hline No & 874 & 52.9 & 1 (ref.) & - & & 1 (ref.) & - & \\
\hline Yes & 138 & 43.5 & 0.69 & $(0.48-0.99)$ & $0.040^{*}$ & 0.61 & $(0.41-0.91)$ & $0.017^{*}$ \\
\hline \multicolumn{9}{|c|}{ Prestroke mRS score } \\
\hline $0-2$ & 872 & 45.8 & 1 (ref.) & - & & 1 (ref.) & - & \\
\hline $3-5$ & 140 & 87.9 & 8.58 & $(5.08-14.50)$ & $<0.001^{*}$ & 6.78 & $(3.96-11.61)$ & $<0.001^{*}$ \\
\hline
\end{tabular}

The test and 95\% CI for the OR were based on the likelihood ratio; a stepwise forward selection method was used to select the variables for multivariate analysis. The threshold for entry was set at $p=0.05$. OR, odds ratio; CI, confidence interval; ref., reference; TIA, transient ischemic attack; mRS, modified Rankin Scale. ${ }^{*} p<0.05$, statistically significant. 


\section{Abnormal Coagulation Was Associated with a Higher Incidence of IHS}

Patients with IHS had significantly higher D-dimer (median 5.8 vs. $0.8 \mu \mathrm{g} / \mathrm{mL} ; p<0.001$ ) and fibrinogen (median 532 vs. $430 \mathrm{mg} / \mathrm{dL} ; p=0.014$ ) levels than patients with OHS (Fig. 1d). There was a high variability in the D-dimer and fibrinogen levels of patients with IHS. This relationship remained significant even when we excluded surgery patients (data not shown). Thus, patients with IHS were more likely to demonstrate hypercoagulable conditions.

\section{Postoperative Ischemic Stroke}

Among 79 patients with IHS, 17 patients (21.5\%) had undergone surgery before stroke onset. The median interval from the surgical procedure to stroke onset was 2 (IQR 1-5) days. The administration of the antithrombotic agent was stopped before surgery in 5 patients $(29.4 \%)$. In 1 patient with $\mathrm{AF}$, warfarin administration was stopped for more than 1 month due to hemorrhagic complications before surgery. In the other patients, the antithrombotic agent was appropriately stopped before surgery and restarted on postoperative day 1 or 2 .

\section{Discussion}

In this study, we document that neoplasms were the most common primary disease in patients with IHS. We found that active cancer and coronary artery disease had a significantly higher prevalence in patients with IHS; however, the most classical stroke risk factors did not differ from those in the OHS patients. The prevalence of stroke of undetermined etiology was higher in patients with IHS, for whom cancer-related ischemic strokes were most common. Furthermore, there was a strong relationship between poorer outcomes and active cancer rather than with the in-hospital onset per se. Taken together, efforts to improve outcomes in patients with IHS should consider the active cancer status.

Cerebrovascular disease is not uncommon in patients with cancer; an autopsy study of 3,426 patients with systemic cancer revealed the presence of cerebrovascular disease in 500 $(14.6 \%)$ patients [14]. In patients with cancer, ischemic stroke is related to poorer outcomes and higher recurrence [15]. Adenocarcinoma, particularly, is associated with recurrent thromboembolism and these events are occasionally diagnosed during a stay in hospital [15]. Importantly, more than half of our patients with IHS had active cancers, with a 59.5\% incidence of adenocarcinoma. These factors are thought to contribute to the poor outcomes in IHS patients. It was recently reported that embolic stroke of undetermined source (ESUS) was not related to long-term outcome [16]. However, a follow-up of ESUS patients, especially those with elevated D-dimer and fibrinogen levels, should be performed with occult cancer in mind because cancer-related ischemic stroke may be a cause of ESUS.

D-dimer and fibrinogen are indicated as potential biomarkers associated with early neurological deterioration following acute stroke in a recent systematic review and metaanalysis [17]. Levels of D-dimer and fibrinogen are elevated in ischemic stroke patients with cancer due to cancer-mediated hypercoagulability $[4,12,13]$; these are also markers of cancer progression [18]. Concurrent high D-dimer levels and multiple vascular territory infarctions are an independent predictor of early recurrent stroke in patients with active cancers [19]. Patients with active cancers who experience arterial ischemic events have a higher rate of death than those with venous thromboembolisms [20].

Higher D-dimer levels are associated with a higher risk of stroke [6]. A recent report indicated that higher D-dimer levels on admission were significantly associated with an increased incidence of ischemic stroke shortly after admission for acute heart failure [3]. This suggests that D-dimer levels may predict short-term ischemic stroke events in patients with acute 
heart failure. Importantly, our study showed that plasma D-dimer levels were higher in patients with IHS. Thus, a high D-dimer level on admission, especially in a patient with active cancer, could be an indicator of increased IHS risk.

Low-molecular-weight heparins and direct oral anticoagulants are recommended for the secondary prevention of cancer-related ischemic stroke; however, the available evidence has come mainly from venous thromboembolism studies or indirect findings (e.g., decreased D-dimer levels or embolic signals) [21]. There is no evidence that prophylactic use of antithrombotic agents for primary prevention of IHS reduces the incidence of cancer-associated ischemic strokes; furthermore, patients with cancer have an increased risk of bleeding [22].

Brain imaging and the initiation of intravenous thrombolysis or endovascular therapy could be delayed after symptoms of ischemic stroke have been recognized in patients with IHS, even with no prehospital phase $[1,2]$. The IHS group had a longer OTE time, in line with other studies. Physicians who initially encounter patients with IHS may be unfamiliar with stroke care. The early detection of patients at a high risk of IHS may enable early referral to a stroke center.

IHS patients tend to have contraindications, such as intravenous thrombolysis [1], and endovascular therapy for major vessel occlusion would improve their outcomes. In recent years, endovascular therapy has drastically improved outcomes in patients who sustain large-vessel ischemic stroke [23]. Furthermore, early endovascular therapy is associated with benefits that may be lost when treatment is delayed [24]. Jung et al. [25] reported that ischemic stroke patients with active cancers who underwent endovascular therapy had good outcomes, similar to those in patients with cardioembolic and large-artery atherosclerosis. If patients at a high risk of IHS are identified at admission, the time from stroke onset to endovascular therapy puncture may shorten. A cancer diagnosis and elevated levels of plasma D-dimer and fibrinogen may help to identify patients at a high risk of IHS.

Interestingly, the multivariate logistic regression analysis revealed that the characteristics common to the IHS patients such as active cancer and prestroke mRS 3-5 (functional dependence) were strongly related to poor outcomes rather than the in-hospital onset per se. The association of older age and female sex with poor outcomes is consistent with recent reports [26]. Statins display neuroprotective effects beyond lipid-lowering in ischemic stroke, but the influence of statin pretreatment on outcomes remains controversial [27]. Postoperative stroke occurred in $0.34 \%$ of a cohort of 36,634 consecutive postoperative patients, and this showed an almost flat distribution over the 30 days after surgery [28]. In hospitalized patients, there are various primary diseases including cancer, and treatment options such as surgery, chemotherapy, and radiation, which can increase the risk of stroke [29]. In this study, there was a high variability in D-dimer and fibrinogen levels in IHS patients; this may be a reflection of the variation in primary diseases and treatment options.

This study has several limitations. First, it was performed in the single stroke center of a university hospital. Patients' backgrounds may vary depending on the hospital in which patients are treated. Second, surgery and other procedures can increase plasma D-dimer and fibrinogen levels; we could not determine why plasma D-dimer and fibrinogen levels were increased in the IHS group. Third, AF-related ischemic stroke may be underdiagnosed because resting and 24-h electrocardiogram are insufficient for detecting AF in most cases.

In conclusion, patients with IHS, especially those with active cancers, had poorer outcomes than patients with OHS. Importantly, elevated plasma levels of D-dimer and fibrinogen may be essential factors for detecting patients who have a high risk of a progression to IHS. 
Yamaguchi et al.: Progression to In-Hospital Ischemic Stroke

\section{Statement of Ethics}

The local ethics committee approved the study and all patients provided informed written consent.

\section{Disclosure Statement}

The authors have no conflicts of interest to declare.

\section{Funding Sources}

There was no funding.

\section{Author Contributions}

I.Y. designed the study and wrote the initial draft of the manuscript. Y.O. contributed to the analysis and statistical interpretation of the data. All other authors contributed the data collection and interpretation and critically reviewed the manuscript. All authors approved the final version of the manuscript and agree to be accountable for all aspects of the work in ensuring that questions related to the accuracy or integrity of any part of the work are appropriately investigated and resolved.

\section{References}

1 Saltman AP, Silver FL, Fang J, Stamplecoski M, Kapral MK. Care and Outcomes of Patients with In-Hospital Stroke. JAMA Neurol. 2015 Jul;72(7):749-55.

2 Schürmann K, Nikoubashman O, Falkenburger B, Tauber SC, Wiesmann M, Schulz JB, et al. Risk profile and treatment options of acute ischemic in-hospital stroke. J Neurol. 2016 Mar;263(3):550-7.

3 Hamatani Y, Nagai T, Nakai M, Nishimura K, Honda Y, Nakano H, et al.; NaDEF Investigators. Elevated Plasma D-Dimer Level Is Associated with Short-Term Risk of Ischemic Stroke in Patients with Acute Heart Failure. Stroke. 2018 Jul;49(7):1737-40.

4 Wang JY, Zhang GJ, Zhuo SX, Wang K, Hu XP, Zhang H, et al. D-dimer $2.785 \mu \mathrm{g} / \mathrm{ml}$ and multiple infarcts $\geq 3$ vascular territories are two characteristics of identifying cancer-associated ischemic stroke patients. Neurol Res. 2018 Nov; 40(11):948-54.

5 Zhang J, Liu L, Tao J, Song Y, Fan Y, Gou M, et al. Prognostic role of early D-dimer level in patients with acute ischemic stroke. PLoS One. 2019 Feb;14(2):e0211458.

6 Zhang J, Song Y, Shan B, He M, Ren Q, Zeng Y, et al. Elevated level of D-dimer increases the risk of stroke. Oncotarget. 2017 Dec;9(2):2208-19.

7 Adams HP Jr, Bendixen BH, Kappelle LJ, Biller J, Love BB, Gordon DL, et al. Classification of subtype of acute ischemic stroke. Definitions for use in a multicenter clinical trial. TOAST. Trial of Org 10172 in Acute Stroke Treatment. Stroke. 1993 Jan;24(1):35-41.

8 WHO International Statistical Classification of Diseases and Related Health Problems - 10th Revision [internet]. Geneva: World Health Organization; 2016 [cited 2019 Apr 5]. Available from: https://icd.who.int/ browse10/2016/en

9 Carbone PP, Kaplan HS, Musshoff K, Smithers DW, Tubiana M. Report of the Committee on Hodgkin's Disease Staging Classification. Cancer Res. 1971 Nov;31(11):1860-1.

10 Sobin LH, Gospodarowicz MK, Wittekind C, editors. TNM Classification of Malignant Tumors. 7th ed. Hoboken (NJ): Wiley-Blackwell; 2009.

11 Lee AY, Levine MN, Baker RI, Bowden C, Kakkar AK, Prins M, et al.; Randomized Comparison of Low-MolecularWeight Heparin versus Oral Anticoagulant Therapy for the Prevention of Recurrent Venous Thromboembolism in Patients with Cancer (CLOT) Investigators. Low-molecular-weight heparin versus a coumarin for the prevention of recurrent venous thromboembolism in patients with cancer. N Engl J Med. 2003 Jul;349(2): 146-53. 
12 Kim K, Lee JH. Risk factors and biomarkers of ischemic stroke in cancer patients. J Stroke. 2014 May; 16(2): 91-6.

13 Selvik HA, Thomassen L, Bjerkreim AT, Næss H. Cancer-Associated Stroke: the Bergen NORSTROKE Study. Cerebrovasc Dis Extra. 2015 Oct;5(3):107-13.

14 Graus F, Rogers LR, Posner JB. Cerebrovascular complications in patients with cancer. Medicine (Baltimore). 1985 Jan;64(1):16-35.

15 Navi BB, Singer S, Merkler AE, Cheng NT, Stone JB, Kamel H, et al. Recurrent thromboembolic events after ischemic stroke in patients with cancer. Neurology. 2014 Jul;83(1):26-33.

16 Tsivgoulis G, Kargiotis O, Katsanos AH, Patousi A, Mavridis D, Tsokani S, et al. Incidence, characteristics and outcomes in patients with embolic stroke of undetermined source: A population-based study. J Neurol Sci. 2019 Jun;401:5-11.

17 Martin AJ, Price CI. A Systematic Review and Meta-Analysis of Molecular Biomarkers Associated with Early Neurological Deterioration Following Acute Stroke. Cerebrovasc Dis. 2018;46(5-6):230-41.

18 Dirix LY, Salgado R, Weytjens R, Colpaert C, Benoy I, Huget P, et al. Plasma fibrin D-dimer levels correlate with tumour volume, progression rate and survival in patients with metastatic breast cancer. Br J Cancer. 2002 Feb; 86(3):389-95.

19 Fujinami J, Ohara T, Kitani-Morii F, Tomii Y, Makita N, Yamada T, et al. Cancer-Associated Hypercoagulation Increases the Risk of Early Recurrent Stroke in Patients with Active Cancer. Cerebrovasc Dis. 2018;46(1-2): 46-51.

20 Brenner B, Bikdeli B, Tzoran I, Madridano O, López-Reyes R, Suriñach JM, et al.; RIETE Investigators. Arterial Ischemic Events Are a Major Complication in Cancer Patients with Venous Thromboembolism. Am J Med. 2018 Sep;131(9):1095-103.

21 Navi BB, Iadecola C. Ischemic stroke in cancer patients: A review of an underappreciated pathology. Ann Neurol. 2018 May;83(5):873-83.

22 Kamphuisen PW, Beyer-WestendorfJ. Bleeding complications during anticoagulant treatment in patients with cancer. Thromb Res. 2014 May;133 Suppl 2:S49-55.

23 Goyal M, Menon BK, van Zwam WH, Dippel DW, Mitchell PJ, Demchuk AM, et al.; HERMES collaborators. Endovascular thrombectomy after large-vessel ischaemic stroke: a meta-analysis of individual patient data from five randomised trials. Lancet. 2016 Apr;387(10029):1723-31.

24 Saver JL, Goyal M, van der Lugt A, Menon BK, Majoie CB, Dippel DW, et al.; HERMES Collaborators. Time to Treatment with Endovascular Thrombectomy and Outcomes from Ischemic Stroke: A Meta-analysis. JAMA. 2016 Sep;316(12):1279-88.

25 Jung S, Jung C, Hyoung Kim J, Se Choi B, Jung Bae Y, Sunwoo L, et al. Procedural and clinical outcomes of endovascular recanalization therapy in patients with cancer-related stroke. Interv Neuroradiol. 2018 Oct;24(5): 520-8.

26 Purroy F, Vena A, Forné C, de Arce AM, Dávalos A, Fuentes B, et al. Age- and Sex-Specific Risk Profiles and In-Hospital Mortality in 13,932 Spanish Stroke Patients. Cerebrovasc Dis. 2019;47(3-4):151-64.

27 Ishikawa H, Wakisaka Y, Matsuo R, Makihara N, Hata J, Kuroda J, et al.; Fukuoka Stroke Registry Investigators. Influence of Statin Pretreatment on Initial Neurological Severity and Short-Term Functional Outcome in Acute Ischemic Stroke Patients: The Fukuoka Stroke Registry. Cerebrovasc Dis. 2016;42(5-6):395-403.

28 Kikura M, Oikawa F, Yamamoto K, Iwamoto T, Tanaka KA, Sato S, et al. Myocardial infarction and cerebrovascular accident following non-cardiac surgery: differences in postoperative temporal distribution and risk factors. J Thromb Haemost. 2008 May;6(5):742-8.

29 Dardiotis E, Aloizou AM, Markoula S, Siokas V, Tsarouhas K, Tzanakakis G, et al. Cancer-associated stroke: Pathophysiology, detection and management (Review) [Review]. Int J Oncol. 2019 Mar;54(3):779-96. 